\title{
Revisión de la Literatura sobre la Toma de Decisiones Éticas en Organizaciones
}

\author{
David A. Díez-Gómez ${ }^{(1) \star}$, Manuel Guillén ${ }^{(2)}$ y María del P. Rodríguez ${ }^{(3)}$ \\ (1) Universidad Nacional de Colombia Sede Manizales, Campus La Nubia, Grupo de Investigación Ethos - Bloque Q, \\ Manizales-Colombia. (e-mail: dadiezgo@unal.edu.co) \\ (2) Universidad de Valencia, Facultad de Economía, Dpto. de Dirección de Empresas, Valencia-España. \\ (e-mail: manuel.guillen@uv.es) \\ (3) Universidad Nacional de Colombia Sede Manizales, Campus La Nubia, Facultad de Ingeniería y Arquitectura, Dpto. \\ de Ingeniería Industrial, Manizales-Colombia. (e-mail: mdrodriguezco@unal.edu.co)
}

* Autor a quien debe estar dirigida la correspondencia

Recibido Jul. 26, 2018; Aceptado Oct. 8, 2018; Versión final Dic. 2, 2018, Publicado Jun. 2019

\section{Resumen}

Este artículo revisa la literatura sobre el tema de toma de decisiones éticas en organizaciones, para establecer sus niveles de integración disciplinar, enfoques teóricos y métodos dominantes. Con ese fin, se combinó la búsqueda sistemática con la narrativa. La sistemática se basó en referencias extraídas de Web of Science (WoS), analizadas mediante la plataforma Tree of Science (ToS) para identificar redes de citación. La narrativa se apoyó en el análisis de referencias complementarias a través del programa Gephi. La literatura se clasificó en tres niveles de diálogo disciplinar: 1) Importación conceptual; 2) Reciprocidad teórica; y 3) Unidad teórica. Se concluye que la escasez de estudios basados en unidad teórica, enfoques integrativos y metodologías mixtas, justifica realizar investigaciones desde una visión más transdisciplinar del conocimiento y la práctica de la toma de decisiones. En esa vía, se propone recurrir al realismo integral complejo como ruta para desarrollar la necesaria visión transdisciplinar de las Decisiones Éticas en Organizaciones.

Palabras clave: ética; ética organizacional; toma de decisiones éticas; transdisciplinario; realismo integral complejo; Tree of Science (ToS).

\section{Literature Review on Ethical Decision Making in Organizations}

\begin{abstract}
This paper reviews the literature on ethical decision making in organizations, to establish its levels of disciplinary integration, as well as its more notorious theoretical and methodological approaches. To that end, systematic and narrative analysis were combined. The systematic review was based on references obtained from the Web of Science (WoS), which were analyzed through the Tree of Science (ToS) platform to identify citation networks. The narrative review was based on the analysis of complementary references through Gephi. The literature was classified into three levels of disciplinary dialogue: 1) Conceptual importation; 2) Theoretical reciprocity; and 3) Theoretical unity. It is concluded that the lack of studies grounded in theoretical unity, integrated and mixed approaches, justifies the development of studies based on a more transdisciplinary vision of decision-making theory and practice. Hence, it is proposed to resort to complex integral realism, as a promising route to develop the required transdisciplinary vision of Ethical Decision Making.
\end{abstract}

Keywords: ethics; organizational ethics; ethical decision making; transdisciplinary; complex integral realism; Tree of Science (ToS). 


\section{INTRODUCCIÓN}

Existe amplio consenso respecto a considerar la investigación sobre Toma de Decisiones Éticas (TDE) en organizaciones como un subcampo académico emergente y en auge dentro del ámbito de la ética organizacional (Tenbrunsel y Smith-Crowe, 2008; Craft, 2012; Lehnert, Park y Singh, 2015; Schwartz, 2016; Lehnert et al., 2016). Tanto la TDE en organizaciones, como la ética organizacional, resultan cada vez más relevantes a nivel mundial, dada la creciente manifestación de prácticas anti-éticas en el mundo de las organizaciones. Desde Watergate y Enron, hasta el reciente caso de corrupción continental de Odebrecht (EI Espectador, 2017), movimientos ciudadanos de todo el mundo demandan un comportamiento más ético por parte de quienes toman decisiones estratégicas en organizaciones del gobierno, el sector privado y la sociedad en general (Transparencia Internacional, 2017). Por ello, es urgente aplicar los hallazgos de la investigación sobre TDE para promover un papel más ético de las organizaciones en la sociedad.

No obstante, el conocimiento sobre TDE enfrenta desafíos teóricos y metodológicos que limitan su potencial impacto social y que vienen heredados del campo más general de la ética organizacional (Boda y Zsolnai, 2016). En el contexto anglosajón, este campo académico comenzó a institucionalizarse en la década de 1980, mediante la creación de revistas especializadas como el Business and Profesional Ethics Journal (1981), el Journal of Business Ethics (1982) y el Business Ethics Quaterly (1991) (Toro y Rodríguez, 2017). Empero, varios autores pioneros (Trevino y Weaver, 1994; Weaver y Trevino, 1994; Donaldson, 1994; Frederick, 1994a, 1994b; Victor y Stephens, 1994) percibieron dificultades para la consolidación de la ética organizacional como un campo de conocimiento claramente identificable. En contravía de esa consolidación, la ética organizacional se ha presentado como dos frentes de investigación basados en aproximaciones disciplinares que, aunque complementarias, se han desarrollado de forma desarticulada.

Por un lado se presenta una aproximación normativa a la ética organizacional, enfocada en establecer cómo debería ser el comportamiento ético en las organizaciones, con base en teorías éticas provenientes de la filosofía y la teología. Desde esta aproximación se estudian preguntas "meta-teóricas" (Trevino y Weaver, 1994) como, por ejemplo, si existe el bien moral, bajo qué teorías puede establecerse ese bien y hasta qué punto estaríamos los seres humanos obligados a ejercerlo. Por otro lado se presenta una aproximación empírica, enfocada en conocer cómo es el comportamiento ético en las organizaciones. Esta perspectiva se fundamenta en ciencias sociales como la administración, la psicología y la sociología. Tales disciplinas se caracterizan por aplicar el método científico, a través de técnicas experimentales, para explicar y -en lo posible- predecir, bajo qué condiciones los individuos y grupos actúan de forma ética o anti-ética en las organizaciones (Trevino y Weaver, 1994). Y más recientemente, la aproximación empírica ha recurrido al uso de diseños cualitativos (Lehnert et al., 2016), a través de los cuales se busca comprender el significado de los comportamientos éticos en las organizaciones, desde el punto de vista de sus propios actores.

Tanto en la investigación empírica cuantitativa como en la cualitativa ha imperado la conceptualización de la TDE como un proceso predominantemente racional (Rogerson et al., 2011). Ello a pesar del llamado de Haidt (2001) a reconocer el importante papel que juegan aspectos no racionales, como las emociones y la intuición, en la dinámica propia de la TDE. Sin embargo, existen iniciativas teóricas de integración entre las imperantes aproximaciones racionalistas y las escasas perspectivas abiertas a aspectos no racionales del proceso de TDE. Tal es el caso del modelo integrativo propuesto por Schwartz (2016).

Asimismo, se ha reconocido la necesidad de integrar las aproximaciones normativas y empíricas, con el fin de generar una más completa comprensión e intervención de la TDE en las organizaciones (Fleming, 1986; Kahn, 1990). Sin embargo, esa integración no parecía estar expresándose en la literatura anglosajona sobre ética organizacional producida tres décadas atrás (Trevino y Weaver, 1994). Caricaturizando un poco la situación, estos últimos autores hacen referencia a una escena típica de la cotidianidad académica de la época. En un evento sobre ética organizacional, un investigador formado en ciencias sociales como la administración o la psicología organizacional, criticaría a los filósofos por no saber nada del mundo de las organizaciones, así como por hablar en un lenguaje incomprensible. En el mismo evento, un filósofo comentaría lo superficiales que son los paneles conformados por miembros de departamentos de administración, en los que -a su parecer- se discuten asuntos superfluos que poco o nada aportan al desarrollo del carácter. Algo comprensible -concluiría el filósofo-, pues los científicos sociales realmente nunca han estudiado la ética. De manera análoga, puede suceder que los investigadores basados en una aproximación racionalista consideren ingenuos a aquellos que siguen un enfoque intuicionista; y viceversa.

Detrás de esta desconexión entre los investigadores de la TDE adscritos a los enfoques normativo, empírico, racionalista e intuicionista, existe un trasfondo epistemológico. Éste puede comprenderse a partir de la taxonomía propuesta por Trevino y Weaver (1994) para clasificar los grados de integración disciplinar expresados en los estudios de ética organizacional: la importación conceptual, la reciprocidad y la unidad teórica. La importación conceptual representa el menor nivel de integración. En este caso, los investigadores 
que siguen una aproximación normativa o empírica (sea esta racionalista o intuicionista), recurren a su contraparte sólo si perciben que la necesitan para, por ejemplo, definir un concepto. Esta sería la tendencia dominante en los comienzos de la ética organizacional, cuando investigadores empíricos tomaban alguna de las teorías de la filosofía moral para establecer una definición operativa del concepto de ética (Rest, 1986; Jones, 1991). La reciprocidad teórica supone, en cambio, un mayor nivel de diálogo disciplinar, en el que los investigadores de uno y otro campo, además de recurrir a conceptos de su contraparte, aprenden el lenguaje asociado a su modus operandi. A este nivel corresponderían aquellas investigaciones que incluyen teorías de naturaleza tanto normativa como empírica-racionalista y empírica-intuicionista, no sólo por necesidad conceptual, sino por una clara intención de establecer relaciones de interdependencia entre distintos tipos de aproximación al conocimiento. Por último, el mayor nivel de diálogo entre aproximaciones normativas y empíricas a la ética organizacional se daría a través de la unidad teórica. Esta última supone no sólo que los académicos normativos aprendan el lenguaje de la investigación empírica (tanto racionalista como intuicionista) y viceversa; sino que, además, se establezca un diálogo global que dé lugar a una "meta-teoría" o nuevo lenguaje holístico de conocimiento.

Los tres niveles descritos guardan correspondencia con las categorías de multi, inter y transdisciplinariedad propuestas por Carvajal (2010). Estas categorías se refieren a grados de interacción entre enfoques disciplinares orientados a producir conocimiento alrededor de un mismo fenómeno. La multidisciplinariedad implica que cada disciplina conserva sus suposiciones, sin permitir que aquellas provenientes de otros ámbitos las enriquezcan. Por su parte, la interdisciplinariedad supone diálogo y colaboración entre disciplinas para producir nuevo conocimiento. Mientras que la transdisciplinariedad implica establecer, además de objetivos comunes de conocimiento, sistemas "totales" o "meta-teóricos", que integren los marcos conceptuales provenientes de distintas disciplinas. Para presentar los resultados de la indagación sobre los niveles de integración disciplinar expresados en la literatura sobre TDE, así como sus implicaciones teóricas, metodológicas y prácticas, este artículo se organiza en cuatro secciones: la primera ilustra el método utilizado, el cual articula la búsqueda sistemática a través de la plataforma web Tree of Science (ToS), con la búsqueda narrativa basada en la teoría de grafos (Robledo et al., 2013; 2014; Zuluaga et al., 2016). La segunda presenta los niveles de diálogo disciplinar, así como los enfoques teóricos y metodológicos presentes en la literatura revisada. La tercera discute las implicaciones de dichos resultados. Y finalmente, en las conclusiones se destacan líneas de investigación relevantes para futuros estudios.

\section{MÉTODO}

Este artículo se suma a otros que han usado la plataforma web ToS como herramienta de búsqueda sistemática para revisar la literatura producida en subcampos de la ética organizacional como la formación en ética en las organizaciones (Toro y Rodríguez, 2017), la evaluación de programas de ética organizacional (Osorio y Rodríguez, 2018) y el liderazgo ético (Correa et al., 2018). Además, se complementa con una búsqueda narrativa (Ferreira et al., 2011), apoyada en el uso del programa de análisis de redes Gephi (Zuluaga et al., 2016). La plataforma web ToS facilita la identificación de literatura relevante sobre un tema académico, así como su clasificación. ToS se alimenta de referencias extraídas a partir de una búsqueda en la base de datos Thomson Reuters, Web of Science - WoS, cuyos resultados son clasificados dentro de tres categorías derivadas de la metáfora de un árbol de conocimiento: raíces, tronco y hojas. Las raíces incluyen las referencias más citadas, clásicas o pioneras. El tronco está conformado por referencias que conectan estudios pioneros con investigación actual, y que contribuyen a la consolidación de diferentes enfoques. Las hojas corresponden a los estudios más recientes, vinculados tanto con las raíces como con el tronco del árbol (Robledo et al., 2013; 2014).

La estructura y los elementos del árbol se establecen a través del análisis de redes de citación. Este análisis consiste en evaluar grupos de artículos o libros, los cuales forman redes al citar referencias y ser citados. Dichas redes son identificadas mediante la aplicación de tres criterios a las referencias bibliográficas: el grado de entrada, el grado de salida, y el grado de intermediación. El grado de entrada se refiere al número de veces que una referencia es citada. El grado de salida se determina por el número de ocasiones en las que una referencia cita a otras dentro de la misma red. Y el grado de intermediación se establece de acuerdo con la probabilidad de que una referencia sea citada por o esté citando a otros dos elementos de la red escogidos al azar. El método ToS se basa en el análisis matemático descrito, para identificar comunidades o grupos de autores que se encuentran dialogando, en el sentido de citarse unos a otros. Por lo tanto, ToS es un método de revisión bibliográfica sistemática, basado en un enfoque cuantitativo. El método ToS ha sido complementado por Zuluaga et al. (2016). Estos autores recurren al programa de análisis de redes Gephi, para visualizar la totalidad de la red de citaciones de la cual se extrae un "árbol" de conocimiento. Dicha visualización facilita la identificación de conglomerados de referencias dentro de las cuales se destacan, a su vez, aquellos autores que son más citados y que más citan referencias dentro de la red. Estos autores suelen estar asociados a enfoques particulares de investigación. 
Siguiendo los parámetros descritos, la consulta en la WoS se realizó el 9 de junio de 2018, con la ecuación de búsqueda: Título=(ethical decision making) Timespan=All years. Databases=SCI-EXPANDED, SSCI, A\&HCl. La búsqueda en la WoS arrojó un total de 665 artículos. Estos fueron exportados a la plataforma ToS, la cual generó un árbol con 80 artículos de diferentes bases de datos, distribuidos en 10 raíces, 10 del tronco y 60 hojas. Con el fin de disminuir el posible grado de arbitrariedad presente en ToS al establecer sólo 10 raíces, 10 componentes del tronco y 60 hojas, Zuluaga et al. (2016) proponen validar y complementar las referencias incluidas en la estructura del árbol. Para realizar la validación, los autores sugieren visualizar toda la red de citaciones derivada de -en este caso- las 665 referencias de las que ToS filtra 80 elementos. Tal visualización se logra ingresando las 665 referencias extraídas de WoS en el programa Gephi. Al hacerlo, se genera una red de 2831 referencias, aglutinadas en ocho conglomerados diferenciados por colores en la Figura 1. El alto número de elementos de la red de Gephi, se debe a que ésta se compone de las 665 referencias de WoS, sumadas a todas las citas incluidas en esas referencias.

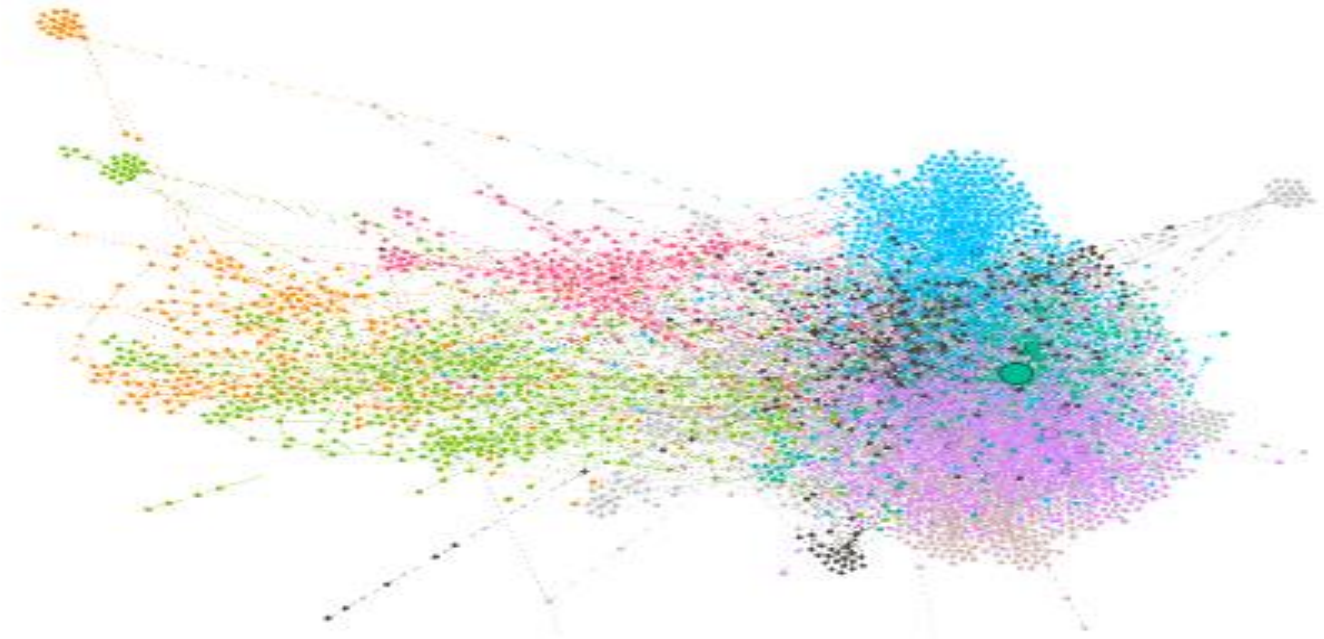

Fig. 1: Estructura de la literatura sobre toma de decisiones éticas arrojada por ToS.

Una vez visualizada la red, se verifica que los artículos más representativos de la misma (en términos del número de veces que son citados y que citan a otras referencias de la red), efectivamente hayan sido incluidos en el árbol de ToS. Esto último se constató. Asimismo, se incluyeron 20 elementos adicionales en el tronco del árbol, completando así un total de 100 referencias seleccionadas. Finalmente, estas 100 referencias fueron analizadas mediante una lógica inductivo-deductiva, cuyos resultados se describen a continuación.

\section{RESULTADOS}

Como se observa en la Tabla 1, la muestra de literatura sobre TDE seleccionada indica que este tema emerge en la década de 1980. Posteriormente, se evidencia la consolidación de una producción más significativa y relativamente regular desde del año 2008 hasta el presente.

Tabla 1: Año de publicación de las referencias consultadas

\begin{tabular}{|l|c|c|c|c|c|c|c|c|c|c|c|c|c|c|}
\hline Año & 1982 & 1983 & 1985 & 1986 & 1991 & 1994 & 2000 & 2001 & 2002 & 2003 & 2004 & 2005 & 2006 & 2007 \\
\hline$N^{\circ}$ Artículos & 1 & 1 & 1 & 2 & 1 & 1 & 1 & 4 & 2 & 3 & 1 & 1 & 3 & 4 \\
\hline Año & 2008 & 2009 & 2010 & 2011 & 2012 & 2013 & 2014 & 2015 & 2016 & 2017 & 2018 & & & \\
\hline$N^{\circ}$ Artículos & 6 & 3 & 7 & 5 & 3 & 10 & 2 & 6 & 15 & 11 & 6 & & & \\
\hline
\end{tabular}

A partir del análisis inductivo de las 100 referencias, se derivaron dos categorías: los conglomerados de Gephi (ver Tabla 2) y los enfoques teóricos (racional e integrado en la Tabla 3). Los conglomerados corresponden a aquellas referencias arrojadas por ToS que pertenecen a cada uno de los nueve subgrupos que Gephi permite identificar de forma automatizada. Por su parte, los enfoques teóricos se definieron a partir de una de las referencias encontradas (Schwartz, 2016), en la cual se diferencian las aproximaciones que conciben la TDE como un proceso principalmente racional y aquellas que, en cambio, abarcan tanto aspectos racionales como emocionales, intuitivos e inconscientes de dicho proceso. 
Tabla 2: Conglomerados de Gephi

\begin{tabular}{|l|c|c|}
\hline Color & № Referencias & Porcentaje \\
\hline Violeta & 35 & $35 \%$ \\
\hline Gris & 19 & $19 \%$ \\
\hline Azul & 16 & $16 \%$ \\
\hline Negro & 11 & $11 \%$ \\
\hline Verde esmeralda & 8 & $8 \%$ \\
\hline Rosa & 4 & $4 \%$ \\
\hline Curuba & 4 & $4 \%$ \\
\hline Verde limón & 2 & $2 \%$ \\
\hline Naranja & 1 & $1 \%$ \\
\hline Total & 100 & $100 \%$ \\
\hline
\end{tabular}

Por otro lado, a partir de una lógica deductiva se analizaron tres aspectos. Primero, el tipo de estudio (ver Tabla 4), siguiendo la diferenciación que plantea Calderón (2005) entre investigación teórica (basada sólo en el pensamiento) y empírico-analítica (que contrasta el pensamiento con la indagación directa de datos reales para validar las construcciones teóricas). A estas dos categorías se añadió la de revisiones bibliográficas, entendidas como aquellas que organizan y analizan estudios realizados por terceros. Aunque las revisiones bibliográficas suelen excluirse de las muestras de otras revisiones de literatura, en este estudio se consideró pertinente incluirlas, pues ellas dan cuenta de las tradiciones académicas más influyentes. El segundo aspecto derivado de una lógica deductica corresponde a los niveles de diálogo disciplinar (ver Tabla 5): la importación conceptual, la reciprocidad y la unidad teórica (Trevino y Weaver, 1994); o, en palabras de Carvajal (2010), la multi, inter y transdisciplinariedad. $Y$ el tercer aspecto fue el método utilizado (cuantitativo, cualitativo 0 mixto) en las investigaciones empírico-analíticas y en las revisiones bibliográficas (Tabla 6).

Tabla 4: Distribución de referencias por tipo de estudio.

Tabla 5: Distribución de referencias según diálogo disciplinar

\begin{tabular}{|l|c|c|}
\hline Tipo de estudio & № Referencias & Porcentaje \\
\hline $\begin{array}{l}\text { Empírico- } \\
\text { Analíticos }\end{array}$ & 74 & $74 \%$ \\
\hline Teóricos & 19 & $19 \%$ \\
\hline $\begin{array}{l}\text { Revisión } \\
\text { bibliográfica }\end{array}$ & 7 & $7 \%$ \\
\hline Total & 100 & $100 \%$ \\
\hline
\end{tabular}

\begin{tabular}{|l|c|c|}
\hline Nivel de diálogo & № Referencias & Porcentaje \\
\hline $\begin{array}{l}\text { Importación conceptual / } \\
\text { multidisciplinariedad }\end{array}$ & 99 & $99 \%$ \\
\hline $\begin{array}{l}\text { Reciprocidad teórica / } \\
\text { interdisciplinariedad }\end{array}$ & 1 & $1 \%$ \\
\hline $\begin{array}{l}\text { Unidad teórica } \\
\text { transdisciplinariedad }\end{array}$ & 0 & $0 \%$ \\
\hline Total & 100 & $100 \%$ \\
\hline
\end{tabular}

Tabla 6: Método según tipo de estudio

\begin{tabular}{|c|c|c|c|c|c|}
\hline Tipo de estudio & $\begin{array}{l}\text { Empírico- } \\
\text { Analítico }\end{array}$ & Teórico & Revisión bibliográfica & № Referencias & Porcentaje \\
\hline $\begin{array}{l}\text { Método } \\
\text { cuantitativo }\end{array}$ & 66 & 0 & 6 & 72 & $72 \%$ \\
\hline $\begin{array}{l}\text { Método } \\
\text { cualitativo }\end{array}$ & 6 & 0 & 1 & 7 & $7 \%$ \\
\hline Método mixto & 2 & 0 & 0 & 2 & $2 \%$ \\
\hline No Aplica & 0 & 19 & 0 & 19 & $19 \%$ \\
\hline Total & 74 & 19 & 7 & 100 & $100 \%$ \\
\hline
\end{tabular}

La discusión de los resultados presentados hasta aquí se organizará a partir de las tres categorías de diálogo disciplinar mencionadas: importación conceptual / multidisciplinariedad, reciprocidad teórica/interdisciplinariedad y unidad teórica / transdisciplinariedad. En cada una de estas categorías se analizarán las tendencias manifiestas en los conglomerados en cuanto a enfoques teóricos, tipos de estudio y métodos utilizados por los investigadores.

\section{DISCUSIÓN}

Como se observa en la Tabla 5 , el $99 \%$ de las 100 referencias se enmarcan en el nivel más bajo de diálogo disciplinar, correspondiente a la importación conceptual (Weaver y Trevino, 1994) o multidisciplinariedad (Carvajal, 2010). Los estudios que componen ese $99 \%$ son en su totalidad realizados por investigadores sociales que recurren a algunas corrientes de la filosofía moral para definir -de manera general- el concepto de ética. Resalta en particular el trabajo de Jones (1991), quien recoge y complementa un conjunto de modelos teóricos pioneros en el estudio de la TDE. Dicho conjunto incluye la propuesta de Rest (1986), según la cual el proceso de TDE se compone de cuatro etapas (consciencia, juicio, intención y comportamiento moral); el modelo de interacción persona-situación que propone Treviño (1986) para analizar la influencia del 
desarrollo moral en el comportamiento ético; y los modelos de Ferrell y Gresham (1985) y Hunt y Vitell (1986) que proponen estudiar el efecto del tipo de razonamiento moral individual (consecuencialista y deontológico) en el proceso de TDE. Este conjunto de modelos son complementados por Jones (1991) mediante el constructo de "intensidad moral". Aunque se suele asumir que este constructo es de naturaleza descriptiva (Ford y Richardson, 1994; Loe et al., 2000; O'Fallon y Butterfield, 2005; Craft, 2012; Lehnert et al., 2015 ), en realidad, se alimenta de planteamientos propios de teorías normativas como el consecuencialismo, la ética del cuidado y el consenso social (Miner y Petocz, 2003).

Además de incluir elementos normativos, el modelo de intensidad moral de Jones (1991) se caracteriza por el predominio de una aproximación racional a la TDE. Como se observa en la Tabla 2 , el $54 \%$ de las referencias revisadas se orientan bajo tal enfoque. Y no porque estudios pioneros como los de Rest (1986) o Jones (1991) consideraran la TDE como un proceso estrictamente racional. De hecho, estos mismos autores reconocían en sus trabajos que el componente racional de la TDE era sólo una parte de un fenómeno más complejo que también abarcaba aspectos emocionales e inconscientes. Sin embargo, especialmente en el caso de Rest, se consideraba adecuado comenzar por estudiar la parte más asequible (la racional) del proceso de TDE. En palabras del autor: "no estoy afirmando que toda decisión moral sea del tipo deliberativo, reflexivo... más bien, hemos decidido comenzar por ese tipo de decisiones por razones de estrategia investigativa" (Rest, 1986: 21).

Pese a la salvedad de Rest, el conglomerado con mayor representación (35\%) dentro de las 100 referencias revisadas (color violeta en la Figura 1 y la Tabla 2), está conformado por 33 estudios orientados bajo un enfoque racional de la TDE. Dentro de estas referencias, cinco de las más representativas (en términos del número de elementos de la red que citan) son las revisiones bibliográficas sobre TDE publicadas en el Journal of Business Ethics (Ford y Richardson, 1994; Loe et al., 2000; O'Fallon y Butterfield, 2005; Craft, 2012; Lehnert et al., 2015). Estas revisiones se enfocan en literatura de naturaleza empírica, a pesar de los reiterativos llamados a la integración de la investigación empírica y normativa publicados, en especial, por el Business Ethics Quarterly (Donaldson, 1994; Frederick, 1994a, 1994b; Trevino y Weaver, 1994; Victor y Stephens, 1994; Weaver y Trevino, 1994). Por otro lado, las cinco revisiones de la literatura sobre TDE publicadas por el Journal of Business Ethics se basan en métodos cuantitativos, al igual que lo hacen 23 de los 25 estudios empírico-analíticos del conglomerado violeta. Mientras que en este mismo conglomerado sólo se presenta una investigación cualitativa y una mixta. Por lo tanto, puede establecerse una relación entre el predominio de un nivel de diálogo disciplinar bajo, un enfoque racionalista y la aplicación de métodos cuantitativos. Esta última tendencia reafirma la crítica de Campbell y Cowton (2015) a la hegemonía de una epistemología positivista en los estudios sobre ética organizacional, heredada especialmente de la escuela de la psicología comportamental estadounidense.

En línea con el paradigma positivista se encuentra la revisión bibliográfica cuantitativa de Trevino et al. (2006), ubicada en el conglomerado azul. No obstante, a pesar de basarse en una aproximación empírica, la revisión de Trevino et al. (2006) trasciende hacia un enfoque integrativo que reconoce las dimensiones racionales y no racionales de la TDE. Dicho enfoque es aplicado por 14 de los 16 estudios del conglomerado azul. Dentro de este conglomerado sobresale la propuesta de Thiel et al. (2012), la cual se alimenta del trabajo pionero en el que Haidt (2001) reivindica el papel central de las emociones y de la intuición en el proceso de TDE. Por otro lado, en la citada revisión de Trevino et al. (2006), los asuntos éticos se definen no sólo como aquellos que ponen en juego consecuencias de alta magnitud (como en el caso de grandes robos y sobornos en el sector público, privado y social), sino también como las situaciones asociadas al cumplimiento de estándares morales mínimos como la honestidad y el respeto de la ley. De igual modo, Trevino et al. (2006) incluyen dentro del marco de los asuntos éticos comportamientos que van más allá de los mínimos morales, como la caridad o la denuncia de actos corruptos.

Una lógica similar a la anterior sigue la única revisión bibliográfica cualitativa encontrada (Tenbrunsel y SmithCrowe, 2008), la cual se ubica en el conglomerado verde esmeralda. Sus autores también siguen una aproximación integrada al proceso de TDE y complementan la importación de conceptos normativos manifiestos en el modelo de intensidad moral de Jones (2001) (asociados al consecuencialismo, la ética del cuidado y la teoría del consenso social) con algunas referencias al principio kantiano de respeto. De este modo, Tenbrunsel y Smith-Crowe asumen los asuntos éticos no sólo como aquellos que ponen en juego consecuencias de alta magnitud, cercanía o niveles de consenso social, sino también como todo conflicto organizacional en el que se ve implicada la dignidad humana. La visión integrativa de Trevino et al. (2006) y Tenbrunsel y Smith-Crowe (2008) también predomina en los conglomerados color gris (con 11 de 19 referencias), azul (14 de 16 referencias), negro (9 de 11 referencias), verde esmeralda (4 de 8 referencias) y rosa (3 de 4). Esto significa que, en medio de la pluralidad de comunidades de investigación de la TDE en organizaciones, se converge hacia el reconocimiento paralelo de las dimensiones racionales, emocionales, intuitivas e inconscientes de dicho fenómeno. 
Tal reconocimiento es evidente en el modelo integrado de TDE propuesto por uno de los trabajos más representativos dentro del conglomerado negro (Schwartz, 2016). Este modelo abarca los elementos consecuencialistas, del cuidado y del consenso social del modelo de Jones (1991), así como los aspectos deontológicos de la propuesta de Tenbrunsel y Smith-Crowe (2008). Adicionalmente, Schwartz (2016) subraya el papel del carácter (asociado a la ética de la virtud) como variable esencial en la comprensión de la TDE. Aún así, Schwartz (2016) cataloga su modelo como descriptivo, quizás en un esfuerzo por adscribir la investigación sobre TDE al ámbito de la ciencia más que al del diálogo con la filosofía o la religión. En todo caso, y a pesar de restringirse a un nivel de diálogo disciplinar bajo, los trabajos de Jones (1991), Treviño et al. (2006), Tenbrunsel y Smith-Crowe (2008) y Schwartz (2016), constituyen referentes teóricos esenciales para aproximarse a la TDE en organizaciones a partir de una visión integrada y no meramente racional sobre este fenómeno. Aún así, tales referentes parecen ser ignorados por miembros de comunidades pequeñas y aisladas en ámbitos particulares de la ética profesional, tales como la consejería, el trabajo social y las ciencias de la información, entre otros (Winston y Bahnaman, 2008; Matusek y Wright, 2010; Yeung et al., 2010). Estos últimos autores proponen valiosas aproximaciones a la TDE en sus propios contextos, pero no trascienden el racionalismo que ya viene superándose en las comunidades con más experiencia en el campo.

Por otro lado, también se presenta el caso de algunos estudios publicados por revistas destacadas en el ámbito de la ética organizacional como el Journal of Business Ethics y el Business Ethics: A European Review, en los que continúan predominando los modelos de corte racional. Ejemplo de ello son tres de los cuatro estudios pertenecientes al conglomerado curuba (Oumlil y Balloun, 2008; Beekun et al., 2010; Fok et al., 2016). Asimismo, se presenta el caso de investigaciones que proponen o siguen modelos integrados, pero que no dialogan con los modelos publicados en las revistas más representativas del campo de la ética organizacional. Tal es el caso del único estudio representante del conglomerado naranja (Berger et al., 2008), publicado en Annals of Internal Medicine.

Si bien la diversidad teórica aporta a la comprensión holística del proceso de TDE, la carencia de unidad conceptual dificulta la consolidación de una tradición que dé lugar a teorías, metodologías y aplicaciones cada vez más efectivas en la promoción de la TDE en las organizaciones. Esta falta de un marco teórico holístico, que vaya más allá de las cuatro etapas propuestas en el modelo pionero de Rest (1986), es reconocida incluso por los autores de las revisiones que clasifican la literatura empírica dentro de esas mismas cuatro etapas (O'Fallon y Butterfield, 2005; Craft, 2012; Lehnert et al., 2015). Pero además, como bien lo señala la única referencia encontrada que llega hasta el nivel de reciprocidad teórica o diálogo interdisciplinar (Miner y Petocz, 2003), el desarrollo teórico, metodológico y aplicativo del campo de investigación sobre TDE, exige mayor conexión entre la literatura empírica existente y la filosofía moral, no sólo para extraer definiciones conceptuales, sino también para complementar el método científico con el pensamiento más abstracto propio del análisis filosófico. En este sentido, el trabajo de Miner y Petocz (2003), ubicado en el conglomerado gris, no se reduce al nivel de la importación conceptual, en el sentido de proponer una definición de la ética a partir de alguna de las teorías morales existentes. Más allá de eso, los autores señalan la necesidad de sustentar toda investigación sobre el proceso de TDE, en una reflexión meta-ética. Esta última, entendida como base lógica necesaria para fundamentar cualquier prescripción y descripción de dicho proceso.

La meta-ética permite a los investigadores establecer la postura que asumen frente a las tres posibles respuestas a la pregunta por la existencia del bien moral: sí existe, no existe o no sabemos si existe. Dentro de esas respuestas se presentan variaciones. Por ejemplo, se puede asumir que el bien moral es una propiedad natural y perceptible a través de los sentidos, aunque no reductible a otros términos naturales (postura realista); o que ese bien moral puede reducirse a otros términos naturales como el placer, la adaptación evolutiva o la utilidad social (postura naturalista); o que el bien moral existe, pero como entidad meta-física, perceptible por medio de la intuición o de la revelación divina (postura no-naturalista). En cualquier caso, los investigadores necesitan la reflexión meta-ética para sustentar en qué criterio se basan para optar por alguna o varias de las teorías éticas normativas existentes. Miner y Petocz (2003) sintetizan esas opciones en cuatro: el consecuencialismo, la deontología, el relativismo normativo y la ética de la virtud. Y así como en la filosofía moral no existe una teoría meta-ética que se imponga definitivamente sobre las demás, tampoco la hay en el ámbito de las teorías normativas.

Dada la inexistencia de una teoría normativa superior, Miner y Petocz sugieren propender por la elaboración de modelos comprehensivos. No obstante, los autores encuentran al menos dos grandes dificultades para formularlos. En primer lugar, que aquellos modelos que pretenden ser de naturaleza descriptiva, en realidad se encuentran permeados por algunas teorías normativas no reconocidas como tales: "los modelos descriptivos del comportamiento ético con frecuencia fallan en el intento de permanecer puramente descriptivos, y se encuentran teñidos de énfasis prescriptivos derivados de una mezcla de supuestos morales subyacentes implícitos y explícitos" (Miner y Petocz, 2003: 15). En segundo lugar, que quienes incluyen perspectivas normativas suelen considerar sólo alguna/s teoría/s, excluyendo, de modo arbitrario, las demás alternativas teóricas existentes. Así, por ejemplo, Miner y Petocz (2003) plantean que aquellos trabajos 
influenciados por la propuesta "empírica" del desarrollo moral de Lawrence Kohlberg (como es el caso de los modelos de Rest, 1986 y Treviño, 1986), en realidad se sustentan en una visión normativa deontológica kantiana. Esto último, teniendo en cuenta que Kohlberg ubica la elección basada en criterios universales de justicia como etapa superior de desarrollo moral. Pero además, desde una mirada feminista como la de Gilligan (1982) -identificada dentro del conglomerado verde limón de la red-, se evidencia un sesgo machista en la teoría del desarrollo moral de Kohlberg, en tanto dicha teoría ubicaría la reflexión racional como criterio superior de juicio moral, por encima de la sensibilidad propia de una ética del cuidado.

Ante la tensión entre el papel de lo racional y lo emocional en el proceso de TDE, Miner y Petocz (2003) abogan por la búsqueda de una integración, considerando que el asunto no es si acaso es más ético basarse en razonamientos abstractos kantianos o en una sensibilidad propia de la ética del cuidado; sino más bien, se trata de cómo combinar adecuadamente ambos aspectos (masculinos y femeninos, racionales y emocionales), según la circunstancia concreta. En concordancia con lo anterior, dichos autores ven más factible adelantar una integración de teorías éticas normativas teniendo como marco global la ética de la virtud. La ventaja de esta vertiente teórica de ética aplicada, es su naturaleza contextual y apertura al análisis paralelo de principios, consecuencias y sensibilidades asociadas a una decisión. Caso distinto al de propuestas de integración normativa que dan prelación al consecuencialismo y a la deontología como referentes morales (Lisman, 1996), dejando las teorías de la virtud y del cuidado como aspectos derivados del principio kantiano de respeto. Esto último es lo que ocurre con el modelo integrativo propuesto por Schwartz (2016), en donde se privilegian las teorías consecuencialista y deontológica. Bajo tal modelo, la ética de la virtud entra en juego sólo en sentido instrumental, pues el carácter se analiza en términos de qué rasgos personales contribuyen o no a que los individuos, grupos $u$ organizaciones, incurran en acciones de graves consecuencias y/o de violación de la dignidad humana. De este modo, se deja de lado el papel de la ética de la virtud en el análisis de asuntos éticos que van más allá de esas acciones, que abarcan el sentido mismo de la vida en general y, más específicamente, de la vida en las organizaciones. Esta última perspectiva sí es considerada, por ejemplo, en la propuesta que plantean Crossan et. al (2013) (conglomerado gris), en el sentido de conectar el modelo de Rest (1986) con una aproximación contemporánea a la teoría aristotélica de la virtud. En la misma línea se encuentra el trabajo de Morales y Cabello (2013), en el cual se propone una definición neo-aristotélico-tomista del concepto de competencias éticas.

Sin embargo, el análisis del papel de la ética de la virtud en la definición de los asuntos éticos -más allá de la concepción del carácter como medio para evitar consecuencias de alta magnitud o de vulneración de la dignididad humana-, supone una discusión ya no sólo científica y filosófica, sino también religiosa (Ananthram y Chan, 2016) y política (Gilligan, 1982). De ahí que se requiera un marco meta-teórico a partir del cual se reconozcan los aportes complementarios de la ciencia, la filosofía, la religión y la política, en tanto ámbitos legítimos para generar conocimiento sobre el proceso de TDE en las organizaciones. Lo anterior nos remite al nivel más alto de diálogo disciplinar: la unidad teórica o transdisciplinar. Respecto a este nivel, no se encontró ninguna referencia en la que se proponga un meta-lenguaje de conocimiento comprehensivo, es decir, un topos a partir del cual puedan integrarse los lenguajes propios de las teorías, métodos y aplicaciones provenientes de aquellas disciplinas científicas, filosóficas, religiosas y políticas relevantes para comprender y mejorar la TDE en las organizaciones. Esta ausencia reitera los llamados a un mayor diálogo transdisciplinar, expresados no sólo durante la primera (Donaldson, 1994; Frederick, 1994a, 1994b; Trevino y Weaver, 1994; Victor y Stephens, 1994; Weaver y Trevino, 1994) y la segunda década (Miner y Petocz, 2003) de existencia del campo de investigación en ética organizacional; sino, incluso, desde el primer encuentro entorno a este campo, realizado en la década de los 70 en los Estados Unidos:

"Si la ética organizacional, en tanto campo académico, va a tener alguna substancia... tendrá que recurrir continuamente no sólo a la filosofía moral y a los estudios sociológicos de los roles y las organizaciones, sino a un número mayor de otros campos... De ahí que pueda resultar fatal si la enseñanza de la ética empresarial es confinada dentro de cualquier disciplina académica" (Maclntyre, 1977: 107).

Cuarenta años después, sigue siendo vigente la invitación al diálogo entre formas de conocimiento diferentes pero complementarias, en torno al estudio de la TDE en organizaciones. Retomando el trabajo de Miner y Petocz (2003), quizás el llamado a la transdisciplinariedad no pueda ser respondido hasta tanto los investigadores en el campo de la ética organizacional no fortalezcan su formación filosófica. Pero este paso requiere no sólo tiempo, sino también disposición para reconocer los límites propios del conocimiento científico. En ese sentido, quizás una pista para avanzar en la ruta hacia la transdisciplinariedad pueda hallarse en el espíritu propio del pensamiento complejo (Morin, 1998). Este último parte de reconocer un elemento esencial en la labor científica: la humilde aceptación de los límites de su conocimiento. 
Teniendo en cuenta esos límites, en este trabajo se sugiere considerar la propuesta realista integral compleja de Marshall (2012a, 2012b; 2016), como marco transdisciplinar para facilitar el diálogo normativo/descriptivo y racionalista/intuicionista en el campo de la investigación sobre TDE. El realismo integral complejo se basa en la articulación entre la perspectiva de la complejidad (Morin, 1998), el realismo crítico (Bhaskar y Hartwig, 2010) y la teoría integral (Wilber, 2006; 2007; 2008). La mirada compleja nos recuerda que la realidad se compone de una amalgama de dimensiones que la ciencia ha pretendido -artificialmente- separar. El realismo crítico postula la premisa ontológica según la cual la realidad humana se compone de múltiples dimensiones, cada una de las cuales puede conocerse mediante distintos métodos. Y la teoría integral propone una matriz que busca reconocer, incluir y organizar todas las dimensiones de la realidad humana en cuatro cuadrantes, correspondientes a objetos de conocimiento y métodos que abarcan pero a la vez trascienden a aquellos propios del predominante ámbito de la ciencia. Adicionalmente, la teoría integral postula los conceptos de líneas y modos para referirse, por un lado, a que todo aspecto de la realidad humana se expresa a nivel racional y emocional al mismo tiempo y, por otro lado, a que existen lógicas masculinas y femeninas cuyo balance es necesario para un adecuado desarrollo de la especie humana.

Una aplicación del lenguaje transdiciplinar que ofrece la perspectiva realista integral compleja al estudio del proceso de TDE en organizaciones (ver Figura 2), permitiría considerar que, antes de pretender medir a través de métodos cuantitativos aquellos comportamientos racionales correspondientes a la dimensión externa de la realidad (cuadrantes superior e inferior derecho), presuponiendo que dichos comportamientos son morales o inmorales, es necesario debatir, a través de métodos argumentativos meta-éticos, qué se está entendiendo por bien moral. Posteriormente, una vez aclarados los supuestos morales de partida, se requiere fundamentar, con base en tales supuestos, aquellas teorías de ética aplicada por las cuales se opta, reconociendo que, dentro de tales teorías, el consecuencialismo (predominantemente materialista) y la deontología (predominantemente racionalista) constituyen sólo dos de cuatro alternativas pertinentes y necesarias para definir los constructos éticos de manera holística: el consecuencialismo, la deontología, el relativismo normativo y la ética de la virtud (Miner y Petocz, 2003).

\begin{tabular}{|c|c|c|}
\hline $\begin{array}{l}\text { Dimensiones } \\
\text { de la realidad } \\
\text { humana }\end{array}$ & INTERIOR & EXTERIOR \\
\hline 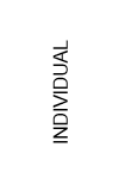 & $\begin{array}{l}\text { Disciplinas: } \\
\text { Filosofia moral } \\
\text { (meta-ética y } \\
\text { ética aplicada) } \\
\text { Objeto: Subjetividad } \\
\text { (Supuestos morales / } \\
\text { Teorías éticas). }\end{array}$ & $\begin{array}{c}\begin{array}{c}\text { Disciplina: } \\
\text { Psicologia }\end{array} \\
\begin{array}{c}\text { Objeto: Objetividad } \\
\text { (Comportamiento } \\
\text { individual). }\end{array}\end{array}$ \\
\hline 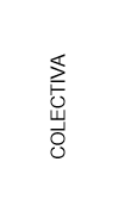 & $\begin{array}{c}\text { Antropologia } \\
\text { cultural } \\
\text { Objeto: Inter-subjetividad } \\
\text { (Valores culturales, } \\
\text { significados } \\
\text { compartidos). }\end{array}$ & $\begin{array}{c}\text { Sociologia, } \\
\text { estudios } \\
\text { criticos, } \\
\text { feminismo } \\
\text { Objeto: Inter-objetividad } \\
\text { (Comportamiento colectivo, } \\
\text { estructuras e instituciones } \\
\text { sociales). }\end{array}$ \\
\hline
\end{tabular}

Fig. 2. Visión realista integral compleja de las disciplinas requeridas para el estudio del proceso de TDE en organizaciones. Adaptación a partir de Marshall (2012), Bhaskar \& Hartwig (2010) y Wilber $(2006,2007,2008)$.

Es necesario conocer y articular los supuestos morales y las propuestas operativas propias de cada una de esas cuatro teorías éticas prescriptivas (cuadrante superior izquierdo en la Figura 2). Luego, ese aparataje conceptual requiere ser comparado con el acervo de valores y prácticas morales propias del contexto cultural específico dentro del cual se pretende investigar el fenómeno de la TDE (cuadrante inferior izquierdo). A partir de esa comparación puede definirse el grado de desconexión o distancia entre el deber ser (perspectiva normativa) y el ser (perspectiva descriptiva). Entonces sí cobra sentido diseñar estrategias tanto a nivel individual (cuadrante superior derecho) como social (cuadrante inferior derecho), orientadas a acercar cada vez más el ser de la TDE en las organizaciones a su deber ser. Asimismo, coincidimos con Miner y Petocz (2003) en cuanto a destacar el papel de la sabiduría práctica como eje de una teorización comprehensiva del proceso de TDE. Tal virtud condensa la habilidad personal y organizacional de traducir conocimientos abstractos -como aquellos propios de las distintas teorías éticas normativas-, en hábitos de mejoramiento continuo evidenciables en la práctica (Bachman et al. 2017). Asimismo, la aplicación de la sabiduría práctica al proceso de TDE, implica la capacidad de balancear polos opuestos, reconociendo sus elementos complementarios (Bachman et al., 2018). Esto último comulga con la demanda feminista de una ética del cuidado (Gilligan, 1982), a partir de la cual se promueva un balance entre aspectos racionales y emocionales, masculinos y femeninos, los cuales aún se encuentran desbalanceados en los valores y en las prácticas culturales de las sociedades actuales. 
La propuesta esbozada puede facilitar la comunicación entre distintas disciplinas de conocimiento (tanto científicas como no científicas). Entendiendo que cada una de esas disciplinas ofrece saberes y estrategias adecuadas para estudiar diferentes - pero complementarias- dimensiones de la TDE. Así, se podrá entablar un diálogo transdiciplinar que contribuya a una mejor TDE en organizaciones, tanto en lo teórico como en lo práctico.

\section{CONCLUSIONES}

Esta revisión sistemática y narrativa de la literatura sobre TDE en organizaciones permitió evidenciar la predominancia de estudios basados en la importación conceptual, la escacez de investigaciones orientadas a partir de la reciprocidad teórica y la ausencia de trabajos desarrollados a partir de una unidad teórica. De ahí que se haga un llamado a realizar investigaciones orientadas desde una visión más transdisciplinar del conocimiento y la práctica de la TDE en organizaciones. Al final, se propone el realismo integral complejo como marco meta-teórico promisorio para desarrollar tal visión, y se destaca el potencial integrativo de la virtud de la sabiduría práctica en la tarea de conectar las dimensiones que componen el proceso de TDE en las organizaciones. Así, de la literatura presentada, su análisis y discusión, se puede concluir lo siguiente:

1) La mayoría de la investigación sobre TDE en organizaciones se basa en un nivel bajo de diálogo disciplinar, limitado a tomar algunas de las definiciones de lo ético presentes dentro de diversos enfoques filosóficos existentes. El único trabajo identificado que trasciende hasta el nivel de la reciprocidad teórica (Miner y Petocz, 2003) hace un llamado, precisamente, a que los investigadores del campo de la ética organizacional complementen su experticia científica con una mayor formación filosófica que les permita enriquecer su capacidad de abstracción. Sin embargo, ninguno de los trabajos revisados responde al temprano llamado de la ética organizacional a rebasar la reciprocidad teórica para formular un lenguaje que permita la comunicación transdisciplinar. De ahí que se haya señalado el realismo integral complejo como marco meta-teórico para responder a ese llamado; y la sabiduría práctica como virtud necesaria para conectar teoría y práctica, así como razones y emociones.

2) Pese a la falta de diálogo transdisciplinar, casi la mitad de las investigaciones revisadas se basan en un enfoque teórico que reconoce ya no sólo las tradicionales dimensiones racionales del proceso de TDE, sino también aquellos aspectos no racionales que lo componen. Aun así, el estudio de tales aspectos demanda complementar el extendido uso de métodos cuantitativos y experimentales, recurriendo en mayor medida a enfoques cualitativos y mixtos, cuya aplicación es todavía marginal en el espectro de las investigaciones sobre TDE en organizaciones.

3) Se identifica como línea de investigación promisoria un mayor avance en el diseño y aplicación de marcos meta-teóricos, basados en una perspectiva realista integral compleja, que abarquen las dimensiones normativas (en sus diversos enfoques filosóficos, religiosos y políticos), empíricas (cuantitativas y cualitativas, racionales y no racionales) de la TDE en organizaciones. Esta incursión es fundamental para consolidar un cuerpo de conocimiento más holístico, consistente y fácil de convertir en aplicaciones útiles para mejorar la TDE en organizaciones de todos los sectores de la sociedad.

4) Finalmente, aunque el carácter exploratorio de la presente investigación supuso restringir el análisis a 100 referencias, esto no impidió establecer una caracterización general de asuntos esenciales del ámbito de conocimiento sobre TDE. De ahí que pueda concluirse que las revisiones de literatura basadas en un análisis sistemático apoyado en la plataforma web ToS, aumentan su efectividad y utilidad al combinarse con el análisis narrativo de referencias extraídas de la red de citaciones generada mediante el programa Gephi. Aun así, esta efectividad puede aumentarse a través de futuros estudios que cubran un espectro más amplio de referencias de la red de citaciones y que continúen incursionando en el uso de herramientas complementarias de análisis de datos. Asimismo, se sugiere contextualizar el análisis de la TDE, a futuro, al ámbito de la literatura y las realidades latinoamericanas.

\section{REFERENCIAS}

Ananthram, S. y C. Chan, Religiosity, Spirituality and Ethical Decision-Making: Perspectives from Executives in Indian Multinational Enterprises, doi: 10.1007/s10490-016-9460-5, Asia Pacific Journal of Management, 33(3), 843-880 (2016)

Bachmann, C., A. Habisch y C. Dierksmeier, Practical Wisdom: Management's No Longer Forgotten Virtue, doi: 10.1007/s10551-016-3417-y, Journal of Business Ethics, First Online, 12 January 2017 (2017)

Bachmann, C., L. Sasse y A. Habisch, Applying the Practical Wisdom Lenses in Decision-Making: An Integrative Approach to Humanistic Management, Humanistic Management Journal, 2(2), 125-150 (2018) 
Beekun, R. I., Y. Stedham, J. W. Westerman y J. H. Yamamura, Effects of Justice and Utilitarianism on Ethical Decision Making: a Crosscultural Examination of Gender Similarities and Differences, doi: 10.1111/j.1467-8608.2010.01600.x, Business Ethics: A European Review, 19(4), 309-325 (2010)

Berger, J. T., E. G. DeRenzo y J. Schwartz, Surrogate decision making: Reconciling Ethical Theory and Clinical Practice, doi: 149/1/48 [pii], Annals of Internal Medicine, 149, 25-48 (2008)

Bhaskar, R. y M. Hartwig, The Formation of Critical Realism, Routledge, Londres, Reino Unido (2010)

Boda, Z. y L. Zsolnai, The Failure of Business Ethics, doi: 10.1108/09574090910954864, Society and Business Review, 11(1), 93-104 (2016)

Calderón, G., Aprender a Investigar Investigando, Editorial UN, Manizales, Colombia (2005)

Campbell, D. y C. Cowton, Method Issues in Business Ethics Research: Finding Credible Answers to Questions that Matter, Business Ethics: A European Review, 24(1), 3-10 (2015)

Carvajal, Y., Interdisciplinariedad: Desafío para la Educación Superior y la Investigación, doi: 1909-2474, Revista Luna Azul, 31(31), 156-169 (2010)

Correa, J.S., M.P. Rodríguez y M. Pantoja, Liderazgo Ético en las Organizaciones: una Revisión de la Literatura, Administer, 32, 57-82 (2018)

Craft, J. L., A Review of the Empirical Ethical Decision-Making Literature: 2004-2011, Journal of Business Ethics, 117(2), 221-259 (2012)

Crossan, M., D. Mazutis y G. Seijts, In Search of Virtue: The Role of Virtues, Values and Character Strengths in Ethical Decision Making, Journal of Business Ethics, 113(4), 567-581 (2013)

Donaldson, T., When Integration Fails: The Logic of Prescription and Description in Business Ethics, doi: 10.2307/3857487, Business Ethics Quarterly, 4(2), 157- 169 (1994)

El Espectador, ¿Qué es el caso Odebrecht?: claves para entender el millonario escándalo de corrupción (periódico digital, febrero 8 de 2017)

Ferreira, I., G.U. González y P. Coello, Systematic Reviews and Meta-Analysis: Scientific Rationale and Interpretation, Revista Española de Cardiología, 64(8), 688-696 (2011)

Ferrell, O. C. y L. G. Gresham, A Contingency Framework for Understanding Ethical Decision Making in Marketing, Journal of Marketing, 49(3), 87-96 (1985)

Fleming, J., A Survey and Critique of Business Ethics Research, en Research in Corporate Social Performance and Policy, por W. Frederick (Ed.), pp. 1-24, JAI Press, Greenwich, Connecticut, Estados Unidos (1986)

Fok, L. Y., D. M. Payne y C. M. Corey, Cultural Values, Utilitarian Orientation, and Ethical Decision Making: A Comparison of U.S. and Puerto Rican Professionals, doi: 10.1007/s10551-014-2426-y, Journal of Business Ethics, 134(2), 263-279 (2016)

Ford, R. C. y W. D Richardson, Ethical Decision Making: A Review of the Empirical Literature, Journal of Business Ethics, 13(3), 205-221(1994)

Frederick, W.C., General Introduction: the Elusive Boundary between Fact and Value, Business Ethics Quarterly, 4(2), 111-112 (1994a)

Frederick, W. C., The Virtual Reality of Fact vs. Value: A Symposium Commentary, doi: 10.2307/3857488, Business Ethics Quarterly, 4(2), 171-173 (1994b)

Gilligan, C., In a Different Voice. Psychological Theory and Women's Development, Harvard University Press, Cambridge, Massachusets, Estados Unidos (1982)

Haidt, J., The Emotional Dog and its Rational Tail: A social Intuitionist Approach to Moral Judgment, Psychological Review, 108(4), 814-834 (2001)

Hunt, S. y S. Vitell, A general Theory of Marketing Ethics, Journal of Macromarketing, 6(1), 5-16 (1986)

Jones, T., Ethical Decision Making by Individuals in Organizations: An Issue-Contingent Model, Academy of Management Review, 16(2), 366-396 (1991)

Kahn, W. A., Toward an Agenda for Business Ethics Research, Academy of Management Review, 15, 311-328 (1990)

Lehnert, K., J. Craft, N. Singh e Y. Park, The Human Experience of Ethics: a Review of a Decade of Qualitative Ethical Decision-Making Research, Business Ethics: A European Review, 25(4), 498-537 (2016) 
Lehnert, K., Y. Park y N. Singh, Research Note and Review of the Empirical Ethical Decision-Making Literature: Boundary Conditions and Extensions, Journal of Business Ethics, 129(1), 195-219 (2015)

Lisman, C., The Circular Integration of Ethics: Theory and Practice, Praeger, Connecticut, Estados Unidos (1996)

Loe, T., L. Ferrell y P. Mansfield, A Review of Empirical Studies Assessing Ethical Decision-Making in Business, Journal of Business Ethics, 25, 185-204 (2000)

Maclntyre, A., Why are the Problems of Business Ethics Insoluble?, en First National Conference on Business Ethics, por M. Hoffman (Ed.), pp. 99-110, Bentley College Press, Waltham, Massachusetts, Estados Unidos (1977)

Mackie, J., The Subjectivity of Values, en Ethics: Inventing Right and Wrong, Penguin, New York, Estados Unidos (1977)

Marshall, P., A complex integral realist perspective: towards a new axial vision, Routledge, New York, USA (2016)

Marshall, P., The Meeting of Two Integrative Metatheories, Journal of Critical Realism, 11(2), 188-214 (2012a)

Marshall, P., Toward an integral realism, Journal of Integral Theory and Practive, 7(4), 1-34 (2012b)

Matusek, J. A., y M. O. Wright, Ethical Dilemmas in Treating Clients with Eating Disorders: A Review and Application of an Integrative Ethical Decision-Making Model, doi: 10.1002/erv.1036, European Eating Disorders Review: The Journal of the Eating Disorders Association, 18(6), 434-452. (2010)

Miner, M., y A. Petocz, Moral theory in ethical decision-making: problems, clarifications and recommendations from a psychological perspective, Journal of Business Ethics, 42(1), 11-25 (2003)

Morales, R. y C. Cabello, The Role of Four Universal Moral Competencies in Ethical Decision-Making, Journal of Business Ethics, 116(4), 717-734 (2013)

Morin, E., Introducción al Pensamiento Complejo, 2ª Ed., Editorial Gedisa, España (1998)

O'Fallon, M. J. y K. D. Butterfield, A Review of the Empirical Ethical Decision-Making Literature: 1996-2003, Journal of Business Ethics, 59, 375-413 (2005)

Osorio, V. y M. del P. Rodríguez, Modelo para la Evaluación de Programas de Ética Organizacional en Universidades, doi: 10.4067/S0718-07642018000100059, Información Tecnológica, 29(1), 59-70 (2018)

Oumlil, A. y J. Balloun, Ethical Decision-Making Differences between American and Moroccan Managers, Journal of Business Ethics, 84(4), 457-478 (2008)

Rest, J., Moral Development: Advances in Research and Theory, Praeger Press, New York, Estados Unidos (1986)

Robledo, S., G. Osorio y C. López, Networking en Pequeña Empresa: una Revisión Bibliográfica utilizando la Teoría de Grafos, Vínculos, 11(2), 6-16 (2014)

Robledo, S., N. Duque e I. Zuluaga, Difusión de Productos a través de Redes Sociales: una Revisión Bibliográfica utilizando la Teoría de Grafos, Respuestas, 18(2), 27-41 (2013)

Rogerson, M.D., M.C. Gottlieb, M.M. Handelsman, S. Knapp y J. Younggren, Nonrational processes in ethical decision making, doi: 10.1037/a0025215, American Psychologist, 66(7), 614-623 (2011)

Schwartz, M., Ethical Decision-Making Theory: An Integrated Approach, Journal of Business Ethics, 139(4), $755-776$ (2016)

Tenbrunsel, A. E. y K. Smith-Crowe, Ethical Decision Making: Where We've Been and Where We're Going, The Academy of Management Annals, 2(1), 545-607 (2008)

Thiel, C. E., Z. Bagdasarov, L. Harkrider, J. F. Johnson y M. D. Mumford, Leader Ethical Decision-Making in Organizations: Strategies for Sensemaking, Journal of Business Ethics, 107(1), 49-64 (2012)

Toro, J. y M. del P. Rodríguez, Formación en Ética en las Organizaciones: Revisión de la Literatura, doi: 10.4067/S071807642017000200018, Información Tecnológica, 28(2), 167-180 (2017)

Transparencia Internacional, Corruption Perception Index (2016)

Trevino, L. K. y G. R. Weaver, Business ETHICS/BUSINESS ethics: ONE FIELD OR TWO? Business Ethics Quarterly, 4(2), 113-129 (1994)

Trevino, L.K., Ethical Decision Making in Organizations: A Person-Situation Interactionist Model, The Academy of Management Review, 11(3), 601-617 (1986)

Victor, B. y C. U. Stephens, Business Ethics: a Synthesis of Normative Philosophy and Empirical Social Science, Business Ethics Quarterly, 4(2), 145-155 (1994) 
Weaver, G. R. y L. K. Trevino, Normative and Empirical Business Ethics: Separation, Marriage of Convenience, or Marriage of Necessity? Business Ethics Quarterly, 4(2), 129-143 (1994)

Wilber, K., Espiritualidad Integral. El nuevo papel de la religión en el mundo actual, Kairoó, Barcelona, España (2007)

Wilber, K., Introduction to Integral Theory and Practice: IOS Basic and the AQAL Map, AQAL: Journal of Integral Theory and Practice, 1(1), 1-40 (2006)

Wilber, K., La visión integral, Introducción al revolucionario enfoque sobre la vida, Dios y el universo, Kairós, Barcelona, España (2008)

Winston, M.D. y S. Bahnaman, Preparation for Ethical Decision-Making: An Analysis of Research in Professional Education, Library \& Information Science Research, 30(3), 222-230 (2008)

Yeung, K., A. Ho, M. Lo y E. Chan, Social Work Ethical Decision Making in an InterDisciplinary Context, British Journal of Social Work, 40(5), 1573-1590 (2010)

Zuluaga, M., S. Robledo y otros cuatro autores, Metabolomics and Pesticides: Systematic Literature Review using Graph Theory for Analysis of References, Nova, 13(25), 7-16 (2016) 
